\title{
Research topic on Pseudomonas aeruginosa, biology, genetics, and host-pathogen interactions
}

\section{Dara W. Frank*}

Department of Microbiology and Molecular Genetics, Center for Infectious Disease Research, Medical College of Wisconsin, Milwaukee, WI, USA

*Correspondence: frankd@mcw.edu

Over the years, Pseudomonas aeruginosa has served as a paradigm for the study of gene expression, metabolism, and pathogenesis. The large genome, which approaches the complexity and size of lower eukaryotes, and an abundance of regulators, facilitates adaptation to almost any environment. During evolution, competition with other prokaryotes and the acquisition of defensive mechanisms to fend off eukaryotic predators have allowed the maintenance of antibiotic-resistance markers, degradative enzymes, and secretion systems, which impact upon human infection. Eleven articles, nine of which are comprehensive reviews, are presented to highlight these topics and provide an up-to-date resource.

\section{GENOMES, PROTEOMES, BIOLOGY}

A perspective on the evolution of genomic diversity is provided in the initial article by Klockgether et al. (2011). The core genome of $P$. aeruginosa is highly conserved and collinearly arranged as a single chromosome. The mosaic structure of the genome is interrupted by the accessory genome being made up of regions of plasticity, integrated islands, transposons, phages, or IS-elements. Comparisons of strains from the same clonal complex, yet unrelated geographic origin, show a low substitution rate. Versatility is related to a combination of a large genome, accessory elements, and a variety of regulatory systems.

Rao et al. (2011) use global proteomic approaches to study the differences between a mucoid and a non-mucoid isolate derived from the same patient. Data from mass spectrometry analyses utilizing isobaric tags and 2D-electrophoresis are correlated with microarray results to provide a snapshot of expression profiles. These techniques indicate that the type 6 secretion (T6S) and the mucoid phenotype are inversely correlated. Important technical considerations make this report a valuable resource for refinements of bacterial proteomics.

\section{METABOLISM, MAINTENANCE OF EXTRACELLULAR MATRICES}

Targeting bacteria-specific metabolic processes has the promise of eradicating not only actively growing cells but also static or slow-growing cells. Arai (2011) provides a review of the terminal oxidases, denitrification enzymes, their roles in aerobic/anaerobic respiration, and control by inter-related regulatory systems. The control of respiratory function is coordinated by RoxSR, which is predicted to sense electron flow through the terminal oxidases or the redox status of the ubiquinone pool. Overall, control/coordination occurs through terminal oxidases, nitric oxide, and the redox status of the respiratory chain.

The metabolic versatility of $P$. aeruginosa is highlighted by its ability to synthesize extracellular matrices in response to environmental conditions. Franklin et al. (2011) provide a comprehensive review of the biosynthetic pathways for three secreted polysaccharides; alginate, Psl, and Pel. The alginate and Pel pathways share characteristics of a lipid-carrier-independent mechanism similar to the biosynthetic complexes of bacterial cellulose and Pga systems. A lipid-carrier-dependent mechanism is consistent with the Psl pathway, comparable to the E. coli capsular synthesis pathways. A framework for additional experimentation is described and questions regarding the biological implications of regulating three pathways are posed.

The plasticity of $P$. aeruginosa in extracellular polysaccharide production is reflected in the ability to produce O-specific antigens that make up the lipopolysaccharides (LPS). Lam et al. (2011) describe the generation of LPS diversity through mechanisms involving horizontal gene transfer, chromosomal insertions, lysogenic conversion, genomic plasticity in the face of in vivo selection, and quorum sensing. The biological implications of the complete LPS, O-specific antigens, core oligosaccharide, and lipid A domains are addressed relevant to flagellar and twitching motility, biofilm formation, adherence, recognition by TLR4, CTFR, host lectin, complement components, and the expression of membrane-associated systems including T3SS and phage receptors. The authors also review the use of LPS components in vaccine formulations.

\section{ANTIBIOTIC RESISTANCE}

Multi-drug resistant $P$. aeruginosa are of clinical concern and Poole (2011) cautions that pan-resistant isolates, treatable only with colistin, are on the rise. The antibiotic-resistance mechanisms addressed include the acquisition of $\beta$-lactamases, particularly the extended-spectrum enzymes, carbapenemases, aminoglycoside-modifying enzymes, and 16S rRNA methylases. Mutational changes causing the upregulation of multi-drug efflux pumps, derepression of $\operatorname{amp} C$, modification of antimicrobial targets, and changes in the outer membrane permeability barrier are described with the propensity of $P$. aeruginosa to exist in vivo and in the environment as slow-growing organisms imbedded in extracellular matrices. Aspects of hypermutation are discussed relative to cystic fibrosis and a lung environment rich in reactiveoxygen species.

In an effort to identify anti-microbial peptides with properties consistent with therapeutic use, Dean et al. (2011) measure the susceptibility of $P$. aeruginosa to several variations of anti-microbial peptides belonging to the cathelicidin family. This research article describes a series of peptides with properties that not only demonstrate toxic effects to the bacteria but also affect bacterial gene expression, twitching motility, and biofilm formation and maintenance. 


\section{PROTEIN SECRETION}

Filloux (2011) elegantly deconstructs all five of the secretion systems that operate in P. aeruginosa. He summarizes the salient features, known protein structures, secreted proteins, or injected proteins in the case of T3SS and T6SS. The common themes include energy requirements, conduit construction, pore formation in the outer membrane, and protein targeting. The perspective from overviewing all systems leads to insights into universal functions and how these systems may have diversified.

Diaz et al. (2011) focus on the T3SS and review the gene regulatory circuitry involved in expression of this secretory/intoxication system. Intrinsic regulation is described with emphasis on the partner-switching mechanisms involving ExsA, ExsC, ExsD, and ExsE. Also, the various environmental signals and stimuli are compiled. The authors provide valuable insights and caveats to interpreting microarray data, concerning strains that overexpress ExsA, host-cell contact, or low-calcium stimuli used to induce T3SS and the requirement for an intact injectisome relative to T3SS regulation.

The transcriptional control of T3SS is complemented by a review of needle-tip proteins that coordinate the assemblage of a translocon into the host-cell membrane. Structural and functional properties of three needle-tip-protein families are compared with emphasis on biophysical and mechanical aspects of regulation including the propagation/amplification of signals to the T3S nanomachine, the control of energy expenditure, protein secretion, and gene regulation within the bacterium as well as feedback regulation by the action of effectors on host-cell physiology. Sato and Frank (2011) point out how the injection process and its understanding provide a wealth of vaccine and therapeutic targets for $P$. aeruginosa.

\section{EPITHELIAL BARRIERS, CELL POLARITY}

Being an opportunistic pathogen, $P$. aeruginosa may be unable to overcome intact epithelial barriers or innate immunity. The interaction of bacteria with intact or wounded epithelium is addressed in the final review article by Engel and Eran (2011). Binding of the bacteria through their pili to host glycans and heparin-sulfate proteoglycans near cell-cell junctions triggers a remodeling of apical surfaces by the recruitment of PI3K resulting in the formation of protrusions. These basolateral-membrane-like protrusions enhance colonization and invasion by this bacterium, suggesting that $P$. aeruginosa subverts epithelial cell regulatory processes that maintain apical-basolateral polarity and prevents wound healing processes.

\section{REFERENCES}

Arai, H. (2011). Regulation and function of versatile aerobic and anaerobic respiratory metabolism in Pseudomonas aeruginosa. Front. Microbiol. 2:103. doi: 10.3389/fmicb.2011.00103

Dean, S. N., Bishop, B. M., and van Hoek, M. L. (2011). Susceptibility of Pseudomonas aeruginosa biofilm to alpha-helical peptides: D-enantiomer of LL-37. Front. Microbiol. 2:128. doi: 10.3389/fmicb. 2011.00128

Diaz, M. R., King, J. M., and Yahr, T. L. (2011). Intrinsic and extrinsic regulation of type III secretion gene expression in Pseudomonas aeruginosa. Front. Microbiol. 2:89. doi: 10.3389/ fmicb.2011.00089

Engel, J., and Eran, Y. (2011). Subversion of mucosal barrier polarity by Pseudomonas aeruginosa. Front.
Microbiol. 2:114.doi: 10.3389/ fmicb.2011.00114

Filloux, A. (2011). Protein secretion systems in Pseudomonas aeruginosa: an essay on diversity, evolution, and function. Front. Microbiol. 2:155.doi: 10.3389/fmicb.2011.00155

Franklin, M. J., Nivens, D. E., Weadge, J. T., and Howell, P. L. (2011). Biosynthesis of the Pseudomonas aeruginosa extracellular polysaccharides, alginate, Pel, and Psl. Front. Microbiol. 2:167. doi: 10.3389/fmicb.2011.00167

Klockgether, J., Cramer, N., Wiehlmann, L., Davenport, C. F., and andTümmler, B. (2011). Pseudomonas aeruginosa genomic structure and diversity. Front. Microbiol. 2:150. doi: 10.3389/ fmicb.2011.00150

Lam, J. S., Taylor, V. L., Islam, S. T., Hao, Y., and Kocíncová, D. (2011). Genetic and functional diversity of Pseudomonas aeruginosa lipopolysaccharide. Front. Microbiol. 2:118. doi: 10.3389/ fmicb. 2011.00118

Poole, K. (2011). Pseudomonas aeruginosa: resistance to the max. Front. Microbiol. 2:65. doi: $10.3389 /$ fmicb. 2011.00065

Rao, J., Damron, F. H., Basler, M. DiGiandomenico, A., Sherman, N. E., Fox, J. W., Mekalanos, J. J., and Goldberg, J. B. (2011). Comparisons of two proteomic analyses of nonmucoid and mucoid Pseudomonas aeruginosa clinical isolates from a cystic fibrosis patient. Front. Microbiol. 2:162.doi: $10.3389 /$ fmicb. 2011.00162

Sato, H., and Frank, D. W. (2011) Multi-functional characteristics of the Pseudomonas aeruginosa type III needle-tip protein, $\mathrm{PcrV}$; comparison to orthologs in other Gram-negative bacteria. Front. Microbiol. 2:142. doi: 10.3389/fmicb.2011.00142

Received: 16 December 2011; accepted: 16 December 2011; published online: 05 January 2012.

Citation: Frank DW (2012) Research topic on Pseudomonas aeruginosa, biol ogy, genetics, and host-pathogen interactions. Front. Microbiol. 3:20. doi: 10.3389/ fmicb.2012.00020

This article was submitted to Frontiers in Cellular and Infection Microbiology, a specialty of Frontiers in Microbiology.

Copyright (C) 2012 Frank. This is an openaccess article distributed under the terms of the Creative Commons Attribution Non Commercial License, which permits non-commercial use, distribution, and reproduction in other forums, provided the original authors and source are credited. 